\title{
Theoretical and Experimental Analysis of Chain Transfer Agents Behaviors in Photopolymer Material
}

\author{
${ }^{1}$ Jinxin Guo, ${ }^{2}$ Michael R. Gleeson, ${ }^{1}$ Shui Liu and ${ }^{1}$ John T. Sheridan ${ }^{*}$ \\ ${ }^{1}$ UCD School of Electrical, Electronic and Mechanical Engineering, \\ UCD Optoelectronic Research Centre, \\ The SFI-Strategic Research Cluster in Solar Energy Conversion, \\ College of Engineering, Mathematical and Physical Sciences, \\ University College Dublin, Belfield, Dublin 4, Ireland. \\ ${ }^{2}$ Department of Computer Science, National University of Ireland Maynooth, \\ Maynooth, Co. Kildare, Republic of Ireland. \\ *Corresponding author: john.sheridan@ucd.ie; Tel: +353 (0)1 7161927
}

\begin{abstract}
The Non-local Photo-Polymerization Driven Diffusion (NPDD) model indicates how a material's performance might be improved, and provides a tool for quantitive comparison of different material compositions and to predict their fundamental limits. In order to reduce the non-locality of polymer chain growth (i.e the non-local response parameter, $\sigma$ ) and to improve the spatial frequency response of a photopolymer material, we introduce the chain transfer agent (CTA). In the literature, extensive studies have been carried out on the improvements of the non-local response modifying by the CTA, sodium formate, in the polyvinyl alcohol-acrylamide (PVA/AA) material. In this article, i) based on the chemical reactions of CTA, we extended the CTA model in the literature; ii) we compare two different CTA materials, sodium formate and 1-mercapto-2-propanol without cross-linker in order to obtain the experimental confirmation of the reduction in the average polymer molecular weight is provided using a diffusion-based holographic technique; iii) we examine the non-local responses of several spatial frequencies with the two CTAs. Using the extended CTA model it is demonstrated that the CTA has the effect of decreasing the average length of the polyacrylamide (PA) chains formed, thus reducing the non-local response parameter, especially, in the high spatial frequency case.
\end{abstract}

Keywords: Holography, photopolymer material, chain transfer agent, non-local response.

\section{INTRODUCTION}

An extended Non-local Photo-polymerization Driven Diffusion (NPDD) model is presented, which includes the effects of: (i) the kinetics of the major photochemical processes; (ii) the temporally and spatially varying photon absorption; (iii) the kinetics of chain transfer; and (iv) the non-local material response. Applying appropriate initial conditions and material parameter values, simulations were carried out using model presented. It shows that by changing the CTA present (concentration and kinetic properties), the average polymer chain length could be controlled. The effects of the non-local response length on a photopolymer material's performance were examined. It also shows that reducing the non-locality of the polymer chains growth will improve the spatial frequency response of the material. ${ }^{1}$ Based on these two results predicted in the model that a controlled decrease in average polymer chain length would result in a lower non-local length, $\sqrt{\sigma}$, and therefore an improved high spatial frequency response. One chemical way to achieve such a reduction in non-local chain growth is to introduce chain transfer agents (CTAs) into a free-radical photopolymer system, causing the production of more, shorter polymer chains. 
In this paper, the validity and generality of the extended NPDD model is examined by applying it to fit experimental data following the inclusion of different concentrations of two types of chain transfer agents, sodium formate (CTA-1) and 1-mercapto-2-propanol (95\%) (CTA-2), in an AA/PVA) based photopolymer material. ${ }^{1-5}$ Model includes effects of CTA now verify experimentally and the best CTA and most appropriate concentration are determined. We note that introducing a CTA into the photopolymer material cause the premature termination of growing macro-radical chains. Their presence therefore leads to a reduction in the average polymer chain length (molecular weight) formed and an increase in the number of individual polymer chains. In order to determine a suitable concentration of CTA, a diffusionbased experimental technique is carried out to examine the effects of including various concentrations of each of the transfer agent tested on the average polymer chain length. Experimental growth curves of unslanted transmission gratings are extracted from the measured diffraction efficiency data, with the most suitable transfer agent concentration being identified by examining the resulting material spatial frequency response. The saturation refractive index modulation for each type of material layer and for a range of spatial frequencies were recorded and discussed.

\section{EXTENDED NPDD MODEL}

\subsection{Photochemical Reactions}

In the case of free radical photo-polymerization systems, the kinetic model describing what takes place involves five main processes, I. initiation, II. propagation, III. termination, IV. inhibition, and V. chain transfer, each of which may involve several the physicochemical reactions. We highlight the major chemical reactions in each process below. ${ }^{6-10}$

\section{Initiation}

During illumination, the reaction between the photosensitiser and the electron donor (co-initiator) leads to the production of initiator radicals, $R^{\bullet}$, which can react with the monomers to produce chain initiators, $M^{\bullet}$.

$$
\begin{gathered}
I \stackrel{h v}{\longrightarrow} R^{\bullet}, \\
R^{\bullet}+M \stackrel{k_{i}}{\longrightarrow} M^{\bullet},
\end{gathered}
$$

where $I$ is the initiator, $h v$ indicates the energy absorbed from a photon, $k_{\mathrm{i}}$ is the chain initiation kinetic constant and $M$ represents a monomer molecule. The absorption process, which occurs during the initiation step, will be discussed in more detail in Section 3.1.

\section{Propagation}

The chain initiator, $M_{1}^{\bullet}$, will attach itself to another monomer molecule, $M$, by addition to the $C=C$ bond yielding a growing polymer radical with an active tip. Through propagation the polymer chain grows, ${ }^{6}$

$$
M_{n}^{\bullet}+M \stackrel{k_{p}}{\longrightarrow} M_{n+1}^{\bullet}
$$

where $k_{\mathrm{p}}$ is the rate constant of propagation and $M_{n}^{\bullet}$ and $M_{n+1}^{\bullet}$ are the growing macro-radical chains of $n$ and (n+1) repeat monomeric units $(n \geq 1)$.

\section{Termination}

Termination can occur in three ways. Two of these, disproportionation and combination, involves two growing macroradicals interacting, i.e., the bimolecular termination mechanism.

$$
\begin{gathered}
M_{n}^{\bullet}+M_{m}^{\bullet} \stackrel{k_{t c}}{\longrightarrow} M_{n+m}, \\
M_{n}^{\bullet}+M_{m}^{\bullet} \stackrel{k_{t d}}{\longrightarrow} M_{n}+M_{m},
\end{gathered}
$$


where $k_{\mathrm{tc}}$ and $k_{\mathrm{td}}$ are the rate constants of combination and disproportionation termination respectively. $M_{n}, M_{m}$ and $M_{n+m}$ represent terminated chains which have no radical tip, i.e., a dead polymer. In this analysis, $k_{\mathrm{tc}}$ and $k_{\mathrm{td}}$ will be treated as a single lumped rate constant, $k_{\mathrm{t}}=k_{\mathrm{tc}}+k_{\mathrm{td}}\left(\mathrm{cm}^{3} \mathrm{~mol}^{-1} \mathrm{~s}^{-1}\right)$, as the mode of termination does not effect the polymerization kinetics. ${ }^{8}$

A third possible termination mechanism involves primary radical termination., ${ }^{8,11}$

$$
M_{n}^{\bullet}+R^{\bullet} \stackrel{k_{t p}}{\longrightarrow} M_{n} R
$$

where $k_{\mathrm{tp}}$ is the rate constant of primary radical termination. In this step, a growing macro-radical chain reacts with a primary radical (initiator radical) leading once again to the production of inactive or dead polymer chains. ${ }^{8}$

\section{Inhibition}

Inhibitors are chemicals which react with the initiating and propagating radical species by rapidly removing or scavenging these radicals. Polymerization is completely halted until they are all consumed. ${ }^{7}$ Several possible inhibitor reaction mechanisms are listed below:

$$
\begin{gathered}
\text { Dye } e^{*}+Z \stackrel{k_{z, D}}{\longrightarrow} \text { LeucoDye }+Z^{*}, \\
R^{\bullet}+Z \stackrel{k_{z, R^{\bullet}}}{\longrightarrow}\left(R+Z^{\bullet} \text {, and/or } R Z^{\bullet}\right), \\
M_{n}^{\bullet}+Z \stackrel{k_{z, M}^{\bullet}}{\longrightarrow}\left(M_{n}+Z^{\bullet} \text {, and } / \text { or } M_{n} Z^{\bullet}\right),
\end{gathered}
$$

where $Z$ is the inhibitor species, e.g., oxygen, Dye ${ }^{*}$ is the excited photosensitiser, $Z^{*}$ is the concentration of singlet oxygen, ${ }^{7,10-12}$ and $k_{z, D}, k_{z, M^{\bullet}}$, and $k_{z, R^{*}}$ are the rate constants of inhibition of the photosensitiser, the macro-radicals and the primary radicals respectively. Inhibition leads to a dead band at the start exposure, i.e., no initial grating formation during exposure. The effects of inhibitors are especially significant when lower exposure intensities are used, for example when large areas must be exposed or short pulses must be used. ${ }^{10}$ In order to further simplify the reaction modelling, we assume that $Z^{*}, R Z^{\bullet}$ and $M_{n} Z^{\bullet}$ do not reinitiate polymerization and that they terminate without regeneration. ${ }^{8,10}$ In addition, $Z^{*}, R-Z^{*}$ and $M_{\mathrm{n}}-Z^{*}$ are assumed to play no further part in grating formation, i.e., no further chain transfer mechanisms involve them. Furthermore, in the sealed material layer, it is assumed that the inhibitors are rapidly consumed at the start of the exposure. ${ }^{13}$

\section{Chain Transfer Mechanism}

Generally, the chain transfer process causes the premature termination of a growing macro-radical chain and arises because of the presence of CTA. ${ }^{7}$ Due to this reaction, a new radical is produced which is referred as a re-initiator. This re-initiator reacts with a monomer molecule to initiate a new growing macro-radical chain. The chain transfer reactions can be written as

$$
\begin{gathered}
M_{n}^{\bullet}+(R I-X) \stackrel{k_{t r, S}}{\longrightarrow}\left(M_{n}-X\right)+R I^{\bullet}, \\
R I^{\bullet}+M \stackrel{k_{r i}}{\longrightarrow}\left(R I-M^{\bullet}\right),
\end{gathered}
$$

where $R I-X$ is the chain transfer agent, $-X$ is the atom or species transferred and $R I^{*}$ is the re-initiator which has a radical tip. $k_{\mathrm{tr}, \mathrm{S}}$ and $k_{\mathrm{ri}}$ are the transfer rate constant to chain-transfer agent and the re-initiation rate constant respectively Due to the premature termination reaction with the chain transfer agent, $R I-X$, the propagating polymer chains will stop growing earlier than they would have if the CTA was not present. We assume that the free radical $R I-M^{\circ}$ produced can be treated as acting chemically identical to a chain initiator $M^{*}$. Therefore the re-initiator, $R I^{*}$, simply initiates a new growing chain with a radical tip $M^{*}$. Thus, while the polymer chains are shortened, the amount of monomer polymerized and the rate of polymerization can remain high. 


\subsubsection{Extended Model}

Based on the analysis in this section, a set of coupled differential equations representing the spatial and temporal evolutions of the material concentrations associated with Eqs. (1) - (5) can be derived in the same manner as described in references [8-10]. For brevity, we only present the equations which are extended by introducing the chain transfer mechanism. a rate equation governing the CTA concentration can be obtain:

$$
\frac{d[C T A(x, t)]}{d t}=\frac{d}{d x}\left\{D_{C T A}(x, t) \frac{d[C T A(x, t)]}{d x}\right\}-k_{t r, S}[C T A(x, t)]\left[M^{\bullet}(x, t)\right] .
$$

It should be noted that, in the following analysis, we only consider chain transfer to chain-transfer agent, i.e., the chain transfer constants for monomer and initiator are assumed negligible. To further simplify the analysis in this paper, we assume that $k_{\mathrm{tr}}=k_{\mathrm{tr}, \mathrm{CTA}}$ and that the CTA diffusion rate, $D_{\mathrm{CTA}}$, is similar to the diffusion rate of monomer, $D_{\mathrm{m}}$, as their molecular weights are similar in the cases examined, i.e., $D_{\text {CTA }} \approx D_{\mathrm{m}}$.

The equation governing the re-initiator concentration is

$$
\frac{d\left[R I^{\bullet}(x, t)\right]}{d t}=k_{t r}[C T A(x, t)]\left[M^{\bullet}(x, t)\right]-k_{r i}\left[R I^{\bullet}(x, t)\right][u(x, t)],
$$

where $R I^{*}$ denotes the re-initiator concentration. The generation term here is the removal term arising in Eq. (5) due to the chain transfer process. Since it is assumed that the initiator radical, $R^{*}$, dominates the primary termination and inhibition processes, we only consider how the re-initiator, $R I^{\circ}$, reacts with the monomer.

Furthermore the chain transfer and re-initiation reactions effect the variation of macro-radical, $\left[M^{\circ}\right]$, and monomer, [u], concentrations. Therefore the equations governing the macro-radical and monomer concentrations must be generalised:

$$
\begin{aligned}
\frac{d\left[M^{\bullet}(x, t)\right]}{d t}=k_{i}\left[R^{\bullet}(x, t)\right][u(x, t)] & +k_{r i}\left[R I^{\bullet}(x, t)\right][u(x, t)]-k_{t}[M \bullet(x, t)]^{2} \\
& \quad-k_{t p}\left[R^{\bullet}(x, t)\right]\left[M^{\bullet}(x, t)\right]-k_{z}[Z(x, t)]\left[M^{\bullet}(x, t)\right]-k_{t r}[C T A(x, t)]\left[M^{\bullet}(x, t)\right],
\end{aligned}
$$

$$
\begin{array}{r}
\frac{d[u(x, t)]}{d t}=\frac{d}{d x}\left\{D_{m}(x, t) \frac{d[u(x, t)]}{d x}\right\}-k_{i}\left[R^{\bullet}(x, t)\right][u(x, t)] \\
\quad-\int_{-\infty}^{\infty} k_{p}\left[M^{\bullet}\left(x^{\prime}, t\right)\right]\left[u\left(x^{\prime}, t\right)\right] G\left(x, x^{\prime}\right) d x^{\prime}-k_{r i}\left[R I^{\bullet}(x, t)\right][u(x, t)] .
\end{array}
$$

where $D_{\mathrm{m}}(x, t)$ represents the monomer diffusion rate, and $G\left(x, x^{\prime}\right)$ is the non-local material spatial response function given $b^{7}$ : 


$$
G\left(x, x^{\prime}\right)=\frac{1}{\sqrt{2 \pi \sigma}} \exp \left[\frac{-\left(x-x^{\prime}\right)^{2}}{2 \sigma}\right]
$$

in which $\sigma$ is the non-local response parameter. The non-local spatial response function represents the effect of initiation at a location $x^{\prime}$ on the amount of monomer polymerized at location $x .{ }^{14} \sqrt{\sigma}$ represents the non-local response length, which is related to the average polymer chain length. ${ }^{15}$

\subsubsection{Polymer Diffusion Model}

It is well known that the average molecular weight of a given polymer has a major effect on the material's physical and mechanical properties. In order to fully characterize a polymer sample, an indication of the distribution of average molecular weights is necessary. We assume a polydisperse polymer system that is made up of polymer chains of similar molecular weights and having similar properties. ${ }^{7}$ We attempt to model the situation where more than one polymer diffusion rate is present in order to characterize such a polydisperse polymer sample. Therefore, we assume that polymer molecule generated during exposure in an AA/PVA material, can be divided into $i$ groups, where $\sum_{i} \varphi_{i}=1$, with $\varphi_{\mathrm{i}}$ being the molecular fraction of the ith group. Each group represents polymer chains of different average lengths having different average molecular weights and different average rate constants of diffusion, $D_{\mathrm{N}, \mathrm{i}}$. Hence, the temporal evolution of the index modulation ${ }^{8-10}$ can be re-expressed as

$$
n_{1}(t)=\frac{\left(n_{\text {dark }}^{2}+2\right)^{2}}{6 n_{\text {dark }}}\left[\phi_{1}^{(m)}(t)\left(\frac{n_{m}^{2}-1}{n_{m}^{2}+2}-\frac{n_{b}^{2}-1}{n_{b}^{2}+2}\right)+\sum \phi_{1}^{(p), i}(t)\left(\frac{n_{p}^{2}-1}{n_{p}^{2}+2}-\frac{n_{b}^{2}-1}{n_{b}^{2}+2}\right)\right],
$$

where $n_{\text {dark }}$ is the refractive index of the photopolymer layer before exposure measured at the replay probe wavelength, $n_{\mathrm{b}}$ is the refractive index of background, which consists of each component without the monomer or polymer presents measured at the replay wavelength, $\phi_{1}^{(m)}(t)$ is the time varying first harmonic volume fraction components of monomer, and $\phi_{1}^{(p), i}(t)$ is the volume fraction contribution to the first polymer harmonic corresponding to the ith polymer group. In this section, we aim to examine and compare the effects of the addition of varying concentrations of chain transfer agents on these physical properties. In order to simplify the model, it is next assumed that the number of groups of average polymer molecular weight can be reduced to two, $i=2$ (small molecular weight and large molecular weight), and that the refractive index of the polymer in each group can be treated as being identical. The equations governing the polymer concentration for each of these polymer groups are,

$$
\begin{gathered}
\frac{d N_{L}(x, t)}{d t}=\varphi_{L} \int_{-\infty}^{\infty} k_{p} M^{\bullet}\left(x^{\prime}, t\right) u\left(x^{\prime}, t\right) G\left(x, x^{\prime}\right) d x^{\prime}-\frac{d}{d x}\left[D_{N, L}(x, t) \frac{d N_{L}(x, t)}{d x}\right], \\
\frac{d N_{S}(x, t)}{d t}=\left(1-\varphi_{L}\right) \int_{-\infty}^{\infty} k_{p} M^{\bullet}\left(x^{\prime}, t\right) u\left(x^{\prime}, t\right) G\left(x, x^{\prime}\right) d x^{\prime}-\frac{d}{d x}\left[D_{N, S}(x, t) \frac{d N_{S}(x, t)}{d x}\right] .
\end{gathered}
$$

Giving the refractive index modulation as,

$$
\begin{aligned}
n_{1}(t)=\frac{\left(n_{\text {dark }}^{2}+2\right)^{2}}{6 n_{\text {dark }}}\left[\phi_{1}^{(m)}(t)\left(\frac{n_{m}^{2}-1}{n_{m}^{2}+2}-\frac{n_{b}^{2}-1}{n_{b}^{2}+2}\right)\right. & \\
& +\phi_{1}^{(p), L}(t)\left(\frac{n_{p}^{2}-1}{n_{p}^{2}+2}-\frac{n_{b}^{2}-1}{n_{b}^{2}+2}\right)+\phi_{1}^{(p), s}(t)\left(\frac{n_{p}^{2}-1}{n_{p}^{2}+2}-\frac{n_{b}^{2}-1}{n_{b}^{2}+2}\right),
\end{aligned}
$$


where $N_{\mathrm{L}}$ and $N_{\mathrm{S}}$ denote the concentrations of the two polymer groups, i.e., Large Polymer and Small Polymer groups. $D_{\mathrm{N}, \mathrm{L}}, D_{\mathrm{N}, \mathrm{S}}, \phi_{1}{ }^{(p), L}(t)$, and $\phi_{1}{ }^{(p), S}(t)$ are the rates of diffusion and the corresponding volume fractions respectively. For simplicity, we introduce a single parameter $\varphi_{\mathrm{L}}$ to determine the time varying fractions of large and small polymer chains, i.e., $\varphi_{\mathrm{L}}$ indicates the percentage of the polymer chains which are Large Polymer. The first-order coupled differential equations in Eqs. (12) and (13), can be derived in the same manner as presented in [8-10]. Combining Eqs. (12) (13) and (14), the temporal evolution of $n_{1}$ can be obtained. Here the initial conditions for $N_{\mathrm{L}}$ and $N_{\mathrm{S}}$ are assumed to be $N_{\mathrm{L}, \mathrm{j} \geq}$ ${ }_{0}(t=0)=N_{\mathrm{S}, \mathrm{j} \geq 0}(t=0)=0$.

\section{EXPERIMENTAL RESULTS}

\subsection{Polymer Diffusion Examination}

In this subsection, we examine the effects of the addition of the chain transfer agents using the model presented above. For each CTA concentration examined, several gratings were recorded at $\Lambda=700 \mathrm{~nm}$ using an exposing intensity of 1 $\mathrm{mW} / \mathrm{cm}^{2}$, at $\lambda=532 \mathrm{~nm}$, employing the holographic set-up described in detail elsewhere. ${ }^{6}$ In all cases the diffraction efficiency of the probe beam $(\lambda=633 \mathrm{~nm})$ was monitored during and post exposure. In order to provide a meaningful comparison, the exposure time was chosen to be $t=50 \mathrm{~s}$ for each case, and equal dosages, $\xi=\sqrt{I_{0}} t$, were delivered. ${ }^{16}$ The diffracted intensity measurement data, having been corrected for Fresnel reflection loses, were then converted into grating refractive index modulations growth curves using Kogelnik’s coupled wave theory. ${ }^{17}$

\begin{tabular}{|c|c|c|c|c|c|}
\hline \multirow{2}{*}{ Composition } & $\begin{array}{c}\text { CTA } \\
\text { Volume } \\
\text { Fraction }\end{array}$ & $\begin{array}{c}\text { Large } \\
\text { Polymer } \\
\text { Fraction, } \varphi_{\mathbf{L}}\end{array}$ & $\begin{array}{c}\mathbf{D}_{\mathbf{N}, \mathbf{S}} \\
\left(\times \mathbf{1 0}^{-13}\right) \\
\mathbf{c m}^{\mathbf{2}} / \mathbf{s}\end{array}$ & $\begin{array}{c}\mathbf{D}_{\mathbf{N}, \mathbf{L}} \\
\left(\times \mathbf{1 0}^{-15}\right) \\
\mathbf{c m}^{\mathbf{2}} / \mathbf{s}\end{array}$ & $\begin{array}{c}\text { MSE } \\
\left(\times \mathbf{1 0}^{-10}\right)\end{array}$ \\
\hline $\begin{array}{c}\text { Standard } \\
\text { (No Cross-linker) }\end{array}$ & - & 0.92 & 1.5 & 1.0 & 1.33 \\
\hline \multirow{3}{*}{ CTA-1 } & a. 0.0152 & 0.88 & 4.0 & 1.5 & 2.20 \\
\cline { 2 - 6 } & b. 0.0225 & 0.79 & 5.2 & 6.5 & 2.18 \\
\cline { 2 - 6 } & c. 0.0300 & 0.62 & 3.5 & 7.5 & 1.90 \\
\hline \multirow{3}{*}{ CTA-2 } & d. 0.0152 & 0.90 & 2.9 & 1.0 & 0.92 \\
\cline { 2 - 6 } & e. 0.0225 & 0.81 & 2.0 & 1.0 & 0.95 \\
\cline { 2 - 6 } & f. 0.0300 & 0.44 & 4.6 & 7.0 & 1.92 \\
\hline
\end{tabular}

Table 1. The rates of polymer diffusion extracted from fitting.

We now examine the effects of the addition of the CTAs. The samples we tested were as follows:

(i) Standard AA/PVA material without cross-linker (no bisacrylamide). (See the row labelled Standard in Table 1).

(ii) Standard material without cross-linker including three different volume fractions of sodium formate (CTA1), (a) 0.015, (b) 0.0225, (c) 0.0300. (See the row labelled CTA-1 in Table 1).

(iii) Standard material without cross-linker and with three volume fractions of 1-mercapto-2-propanol (CTA-2), (d) 0.015, (e) 0.0225, (f) 0.0300. (See the row labelled CTA-2 in Table 1).

The purpose of the cross-linker (bisacrylamide) is to bind the polyacrylamide chains, which are formed during the recording process. This helps to reduce the mobility of the polymer chains and ensures the stability of the grating formed. Removing the cross-linker will allow the polyacrylamide chains to diffuse out of the bright regions of the 
recorded fringe pattern, resulting in a decay of the grating refractive index modulation with time. Another point to note is that, when the cross-linker is not included in the material composition, the volume fractions of the other components change. For this reason, an equivalent small volume of monomer is added to compensate these changes, thus maintaining the same volume fractions of the other components.

The diffusion model was applied to examine the polymer diffusion. A least square algorithm was used to fit the model to the refractive index modulation curves. Using this technique, values for the diffusion rates of, the Large Polymer and Small Polymer molecular weight groups and the Large Polymer fraction, $\varphi_{\mathrm{L}}$, were extracted and are presented in Table 1. The corresponding MSE values for the fits are also provided to indicate the quality of the fits to the experimental data. As can be clearly observed from Table 3, an increase in the volume fraction of chain transfer agent leads to a decrease in the Large Polymer fraction, $\varphi_{\mathrm{L}}$. In all cases, the rate constant of diffusion $D_{\mathrm{N}, \mathrm{L}}$ are in the range of $10^{-15} \mathrm{~cm}^{2} / \mathrm{s}$ and all $D_{\mathrm{N}, \mathrm{S}}$ values are in the range of $10^{-13} \mathrm{~cm}^{2} / \mathrm{s}$. These values indicate that the number of small polymer chains become greater as the volume fraction of chain transfer agent present increases, and thus importantly that the inclusion of the CTAs increase the number of small polymer chains. For CTA-1, the $\varphi_{\mathrm{L}}$ values decrease from 0.88 to 0.62 with the increase in volume fraction of sodium formate from 0.0152 to 0.03 . This corresponds to approximately a $30 \%$ reduction in the fraction of long polymer chains formed, when compared to the standard material composition case. For the case of the addition of CTA-2-(f), the smallest value obtained for $\varphi_{\mathrm{L}}$, was 0.44 , when a volume fraction of 0.03 was added. Experimentally, it should be noted that there is a sufficiently large reduction in the average molecular weight to allow the effects of the addition of CTA on the average polymer chain length.

From the results obtained and presented in Table 1, the rate of decay of refractive index modulation obtained for case CTA-2-(f) is faster than the values obtained for all the other samples examined. This indicates that the material composition of case CTA-2-(f) produces polymer chains with the lowest average molecular weight and therefore having the shortest average polymer chain length, and hence should provide the largest reduction in the non-local response parameter. As great care was taken to accurately estimate the $\varphi_{\mathrm{L}}$, values, and since the results obtained are based on averages produced using many reproducible data sets, the effect of the reduction in the non-local response should be most prominent in this material composition corresponding to case CTA-2-(f).

As the analysis indicated in reference [1], the CTA has little effect on the average refractive index or on the rate of monomer diffusion in the layer. Based on the experimental results and analysis above, it appears as though the most suitable CTA volume fractions to reduce the non-local response length are 0.03 for each of the CTAs examined. We therefore proceed to examine these material compositions, experimentally producing the spatial frequency response for each.

\subsection{Spatial Frequency Response}

In this subsection, we aim to demonstrate and compare the effects of the CTAs on the spatial frequency response of the materials under examination. Following the analysis presented in this paper, we show that the demonstrated reduction in average polymer chain length reported in Subsection 3.2 does indeed reduce the non-local chain length variance, $\sqrt{\sigma}$. In order to experimentally confirm this hypothesis, the growth curves and the saturation values of refractive index modulation, $n_{1}$, for each photopolymer material composition, for spatial frequencies from 500 to 3000 lines $/ \mathrm{mm}$, are measured and compared. 6,16

In the same manner as described in references [10,13], the absorption parameters were obtained from the fits to the normalised transmitted recording beams captured during the recording of the holographic gratings. These parameters determine the absorption behaviour of the materials and therefore the generation of the initiator radicals. The values estimated are, $\varepsilon=1.43 \times 10^{8} \mathrm{~cm}^{2} / \mathrm{mol}, \phi=0.018$, and $T_{\mathrm{sf}}=0.76$, and are all in agree with previous estimated in the literature. $^{1,9-11,13}$ The exposing fringe visibility was $V=1$ and the relationship assumed between rate constants used were $k_{\mathrm{p}}=k_{\mathrm{i}}$, and $k_{\mathrm{tp}}=k_{\mathrm{t}} \times 10 .^{8-11}$

In order to accurately represent the growth curves of refractive index modulation obtained for each spatial frequency and each material composition, an average growth curves with appropriate error bars were generated from all the repeated experimental data sets and were is used during the fitting procedure. Applying the extended NPDD model developed, the experimental growth curve data was then fit using a least squares algorithm, in which the Mean Square Error (MSE) 


\begin{tabular}{|c|c|c|c|c|}
\hline \multirow{3}{*}{$\begin{array}{l}\text { between the } \\
\text { model and the } \\
\text { was minimized to } \\
\text { material } \\
\text { order to carry out } \\
\text { search ranges of } \\
\text { values based on } \\
\text { the literature were }\end{array}$} & SF (lines/mm) & $\underset{\left[n_{1}^{\text {sat }} \times 10^{-3}\right]}{\text { Stand. }}$ & $\begin{array}{c}\text { CTA-1 } \\
{\left[n_{1}^{\text {sat }} \times 10^{-3}\right]}\end{array}$ & $\underset{\left[n_{1}{ }^{\text {sat }} \times 10^{-3}\right]}{\text { CTA-2 }}$ \\
\hline & 500 & 2.07 & 1.90 & 1.97 \\
\hline & 1000 & 2.20 & 2.18 & 2.09 \\
\hline \multirow{4}{*}{$\begin{array}{l}\text { The model was } \\
\text { analyse the } n_{1} \\
\text { curves, for each } \\
\text { spatial frequency. } \\
\text { monomer }\end{array}$} & 1428 & 2.36 & 2.39 & 2.38 \\
\hline & 2000 & 1.97 & 2.12 & 2.23 \\
\hline & 2500 & 1.56 & 1.87 & 2.03 \\
\hline & 3000 & 1.38 & 1.60 & 1.68 \\
\hline \multirow{2}{*}{$\begin{array}{ll}D_{\mathrm{m} 0}, & \text { the } \\
\text { constant, } \quad k_{\mathrm{p}}, & \text { the } \\
\text { bimolecular } & \end{array}$} & $\sqrt{\sigma}(\mathbf{n m})$ & $61.3 \pm 6.7$ & $47.6 \pm 3.4$ & $39.8 \pm 2.2$ \\
\hline & MSE $\left(\times 10^{-10}\right)$ & $1.59 \pm 1.13$ & $2.41 \pm 1.24$ & $2.18 \pm 3.35$ \\
\hline
\end{tabular}

prediction of the experimental data extract key parameters. In the fitting process, typical parameter data presented in used. ${ }^{1,8-11,18-19}$ then applied to temporal growth material at each In this way the diffusion constant propagation rate initial rate, $k_{\mathrm{i}}$, termination rate constant, $k_{\mathrm{t}}$, the primary

termination rate, $k_{\mathrm{tp}}$, the chain transfer rate, $k_{\mathrm{tr}}$, the re-initiation rate, $k_{\mathrm{ri}}$, and the non-local response length, $\sqrt{\sigma}$, were all estimated. The parameters estimated for the compositions studied are presented in Table 2.

Table 7: Estimations for spatial frequencies in standard AA/PVA (Stand.), standard with sodium formate (CTA-1) and 1-mercapto-2-propanol (CTA-2) material.

\section{CONCLUSION AND ANALYSIS}

In this paper, an extended NPDD model, which includes the effect of a chain transfer agent in the kinetics of free-radical photo-polymerization, and also includes most of the other major photochemical reactions and mass transport effects occurring during the holographic grating formation, have been experimentally examined. The prediction of this model, that a decrease in the non-local response length will cause an improvement in the high spatial frequency response of the photopolymer material, is explored. A number of material layers made using various concentrations of two CTAs, i.e., sodium formate and 1-mercapto-2-propanol, were examined by recording growth curves at a range of spatial frequencies. These curves were then numerically fit with the proposed NPDD model and key parameters were extracted to quantify the effects on the non-local response length and hence the material's spatial frequency response.

To examine the effect of the addition of transfer agent on the average polymer chain length, a diffusion-based technique was developed and the results presented. It was assumed for simplicity that the polymer chains formed during recording 
could be grouped into two subsets having different average molecular weights in order to characterise polymer chain diffusion in a non-cross-linked photopolymer system. In this way, a comparison of polymer diffusion rates in standard material and material containing different types and different concentrations of chain transfer agents was obtained. In this way, the most appropriate volume fraction for each transfer agent to be used was explored.

In order to experimentally validate the prediction of the model, it was applied to fit experimental data in three types of materials and material parameters estimated. The improvement in spatial frequency response of an AA/PVA photopolymer through the addition of a chain transfer agent has been demonstrated. This arise is due to the effect of transfer agent in decreasing the average length of the polymer chains formed, which contribute to the increased localization of the polymerization. The most effective material combination used resulted in a reduction in the non-local parameter, from $61 \mathrm{~nm}$ to $41 \mathrm{~nm}$. Comparing the reduction of $\sqrt{\sigma}$ achieved by the two types of transfer agents, 1 mercapto-2-propanol, has been shown to be better than sodium formate. Furthermore other key material kinetics parameters were extracted by fitting the experimental data, including the rate constants of chain transfer and re-initiation, thus the kinetic behaviour of transfer agents, have been quantified. The prediction and results in these series of papers indicate that a particular type of CTA with a higher rate constant of chain transfer will produce a lower average polymer chain length and therefore a reduction in the non-local response length. An appropriate re-initiation rate (efficiency) is also required. The low MSE values achieved and good general agreement between parameters extracted using different experimental data sets indicate the consistent quality of predictions of the model. Future work will include the derivation of a more accurate formula expressing the relationship between the number-average degree of polymerization and the non-local response length, $\sqrt{\sigma}$.

\section{ACKNOWLEDGMENTS}

One of the authors (JG) currently holds an IRCSET Government of Ireland Postgraduate Scholarship in Science, Engineering and Technology, while another author (MRG) currently holds a Government of Ireland Post-doctoral Scholarship in Science, Engineering and Technology, both granted by the Irish Research Council for Science, Engineering and Technology. We would also like to acknowledge the support of Enterprise Ireland and Science Foundation Ireland under the National Development Fund.

\section{REFERENCES}

[1] M. R. Gleeson, D. Sabol, S. Liu, C. E. Close, J. V. Kelly, and J. T. Sheridan, "Improvement of the spatial frequency response of photopolymer materials by modifying polymer chain length,” J. Opt. Soc. Am. B 25(3), 396-406, (2008).

[2] M. Fevola, R. Hester, and C. McCormack, "Molecular weight control of polyacyrlamide with Sodium Formate as a Chain-Transfer Agent: Characterization via size exclusion chromatography/ multi-angle laser light scattering and determination of Chain-Transfer Constant,” J. Polym. Sci., Part A: Poly. Chem. 41, 560-568, (2003).

[3] A. Valdebenito and M. V. Encinas, "Thiophenols as Chain Transfer Agents in the Polymerization of Vinyl Monomers,” Polymer, 46, 10658-10662, (2005).

[4] F. Karasu, N. Arsu, and Y. Yagci, "2-Mercapto Thioxanthone as a Chain Transfer Agent in Free-Radical Polymerization” J. Appl. Poly. Sci. 103, 3766-3770, (2007).

[5] http://www.arkema-inc.com/index.cfm.

[6] J. R. Lawrence, F. T. O'Neill, and J. T. Sheridan, "Photopolymer holographic recording material," Optik 112(10), 449-463, (2001).

[7] G. Odian, Principles of Polymerization $4^{\text {th }}$ Edition, (Wiley, New York, 1991).

[8] M. R. Gleeson and J. T. Sheridan, "Non-local photo-polymerization kinetics including multiple termination mechanisms and dark reactions: Part I. Modelling,” J. Opt. Soc. Am. B 26(9), 1736-1745, (2009).

[9] M. R. Gleeson, S. Liu, R. R. McLeod, and J. T. Sheridan, "Non-local photo-polymerization kinetics including multiple termination mechanisms and dark reactions: Part II. Experimental Validation,” J. Opt. Soc. Am. B 26(9), 1746-1754, (2009). 
[10] M. R. Gleeson, S. Liu, J. Guo and J. T. Sheridan, "Non-Local photo-polymerization kinetics including multiple termination mechanisms and dark reactions: Part III. Primary Radical Generation and Inhibition,” J. Opt. Soc. Am. B accepted, (2010).

[11] S. Liu, M. R. Gleeson, J. Guo and J. T. Sheridan, “Optical characterization of photopolymers materials: Theoretical and experimental examination of primary radical generation,” Appl. Phys. B 100, 559-569 (2010).

[12] A. Fimia, N. Lopez, F. Mateos, R. Sastre, J. Pineda, and F. Amat-Guerri, "Elimination of oxygen inhibition in photopolymer systems used as holographic recording materials,” J. Mod. Opt. 40, No. 4, 699-706, (1993).

[13] S. Liu, M. R. Gleeson, J. Guo, and J. T. Sheridan, "High intensity response of photopolymer materials for holographic grating formation,” Macromolecules 43, 9462-9472, (2010).

[14] J. T. Sheridan and J. R. Lawrence, "Nonlocal response diffusion model of holographic recording in photopolymer,” Journal of the Optical Society of America A 17(6), 1108-1114, (2000).

[15] M. Doi, Introduction to Polymer Physics (Oxford, 1997), pp. 1-8.

[16] M. R. Gleeson, J. V. Kelly, and J. T. Sheridan, "Modelling the photochemical effects present during holographic grating formation in photopolymer materials,” J. Appl. Phys. 102, 1-9 (2007).

[17] H. Kogelnik, "Coupled wave theory for thick holographic gratings,” Bell Sys. Tech. J. 48, 2909-2947, (1969).

[18] S. Wu, E. N. Glytsis, "Holographic grating formation in photopolymers: analysis and experimental results based on a nonlocal diffusion model and rigorous coupled-wave analysis,” J. Opt. Soc. Am. B 20, 1177-1188, (2003).

[19] J. Brandrup, E. H. Immergut, and E. A. Grulke, Polymer Handbook $4^{\text {th }}$ Edition (Wiley, 1999). 